\title{
Antibiotic Resistance Pattern of Methicillin Resistant Staphylococcus aureus Isolated From Clinical Specimens
}

\author{
Salah Uddin Khan ${ }^{1}$, Mahmood Ahmed Chowdhury ${ }^{2}$, Md. Abdul Hakim ${ }^{3}$ \\ ${ }^{I}$ Department of Microbiology, University of Chittagong, Bangladesh \\ ${ }^{2}$ Department of Microbiology, Chattagram Maa Shishu O General Hospital, Chittagong, Bangladesh \\ ${ }^{3}$ Department of Microbiology, University of Chittagong , Bangladesh
}

\begin{abstract}
Methicillin Resistant S. aureus poses serious threat to human health since the mortality and morbidity rate due to $S$. aureus infection is significant. In the present study, a total of 37 S. aureus strains isolated from 1078 clinical were tested against various antibiotics. Antibiotic susceptibility of the isolated $S$. aureus was carried out using disk diffusion method. The MIC and MBC of different antibiotics were determined by tube dilution assay and disk diffusion technique. The maximum prescribed antibiotic groups were cephalosporins and penicillins. The most resistant group was penicillin followed by aminoglycoside, cephalosporins and quinolones. The order of resistance of S. aureus against various antibiotics was ampicillin (92.10\%), cloxacillin (61.29\%), cefradine (60\%), ciprofloxacin (59.35\%), gentamicin (58.06\%). There was no resistant isolate against vancomycin; however, four isolates (10.52\%) had shown intermediate sensitivity. MIC and MBC of five major groups of antibiotics namely, penicillins, cephalosporins, aminoglycosides, quinolones and vancomycin were also determined. In-vitro study of MBC of the five groups of antibiotics showed 2 to 3 fold two-fold dilution higher value than in MIC of each group of antibiotic. Only MBC of vancomycin was in the range of 1 to 2 two-fold dilutions higher than their MICs.
\end{abstract}

Keywords - Antibiotic resistance, MRSA, MIC, MBC, Staphylococcus aureus

\section{Introduction}

Multidrug resistance is now a very common norm in S. aureus. The mortality of $S$. aureus bacteremia remains approximately $20-40 \%$ despite the availability of effective antimicrobials, which indicates the propensity of $S$. aureus to develop resistance. Some strains that infect hospitalized patients are resistant to most of the antibiotics used to treat infections, vancomycin being the only remaining drug to which resistance has not developed [1]. There are also reports of MRSA strains with less susceptibility toward Vancomycin [2]. Some identified genes to be the cause of such resistance. Staphylococcus cassette chromosome mec, encodes methicillin resistance in Staphylococcus aureus [3]. S. aureus resistance to penicillin is mediated by blaZ, the gene that encodes $\beta$-lactamase. Methicillin resistance requires the presence of the chromosomally localized $m e c A$ gene. The cause behind the resistance of quinolones to be the stepwise acquisition of chromosomal mutations. Vancomycin resistance has resulted from the probable conjugal transfer of the vanA operon from a vancomycin-resistant $E$. faecalis ${ }^{[4]}$. The study was thus conducted to observe the pattern of antibiotic resistance of $S$. aureus isolated from clinical samples.

\section{Material and Methods}

A total of 1078 samples received in the microbiology laboratory of Chattagram Maa Shishu O General Hospital, which include blood, pus and miscellaneous samples (swabs/sputum/body fluids).

\subsection{Sample Processing}

\subsubsection{Blood}

Blood samples were collected from patients visiting OPD (Out Patients Department), different wards (IDP) and were brought to the pathology laboratory. Freshly drawn, 3-5 mL blood was immediately transferred to $50 \mathrm{~mL}$ of Tryptone Soya broth (CM0129-OXOID), and incubated at $37^{\circ} \mathrm{C}$ for 24 hours. Then sub-cultured on Blood Agar (CM 0055- OXOID), Chocolate agar (by heating Blood Agar at $63^{\circ} \mathrm{C}$ ) and MacConkey Agar (CM 0007-OXOID) plates, and incubated for 24 hours at $37^{\circ} \mathrm{C}$.

\subsubsection{Pus}

Pus samples were plated directly onto Blood Agar (CM 0055- OXOID), Chocolate agar (by heating Blood Agar at $63^{\circ} \mathrm{C}$ ) and MacConkey Agar (CM 0007-OXOID), and incubated for 24 hours at $37^{\circ} \mathrm{C}$. 


\subsubsection{Miscellaneous Samples}

Body fluids, sputum and swab samples were cultured on Blood agar, MacConkey agar and Chocolate agar, respectively and then incubated for 24 to 48 hours at $37^{\circ} \mathrm{C}$.

\subsection{Sub-Culturing and Maintenance of Bacterial Isolates}

Randomly selected isolates were sub-cultured and preserved for further studies. For this purpose mid exponential phase bacterial isolates were inoculated into 10\% non-fat Skim Milk (DIFCO) and maintained at $4^{\circ} \mathrm{C}$. In this condition, bacterial culture can be preserved for as long as one month.

\subsection{Identification}

All of the isolates were identified by morphological and biochemical properties as outlined in the Bergey's Manual of Determinative Bacteriology (9th edition). The isolates were additionally investigated by PCR amplification of species specific parts of the gene encoding the 23S rRNA with the oligonucleotide primers [5]

\subsection{Antibiotic Susceptibility Test}

The resistance pattern among these isolates against different groups of antibiotics, which are commonly in use, was determined by disk diffusion technique [6]. Commercially available disks were applied on Mueller Hinton Agar (CM0337-OXOID) as recommended by the manufacturer.

\subsection{Determination of MIC and MBC}

Agar dilution method was used to determine the minimum inhibitory concentrations (MIC), or the lowest concentration of antimicrobial agent required to inhibit the microorganism. Serial two fold dilution concentrations of ampicillin, cefradine, gentamicin, ciprofloxacin and vancomycin active pharmaceutical ingredient (API) and their respective brands were made (TABLE 1) and tested against S. aureus from the clinical specimens. Inoculum of MIC isolates and control S. aureus were subcultured on blood agar plates and incubated overnight at $35^{\circ} \mathrm{C}$.

\section{Susceptibility Pattern of $S$. aureus}

\section{Results and Discussion}

The susceptibility of 38 strains of Staphylococcus aureus were assessed against various antimicrobial agents by disk diffusion method. Most of the $S$. aureus were multi drug resistant and Vancomycin was found to be most effective drug against these clinical isolates. There was no resistant isolate against vancomycin and only $4(10.52 \%)$ isolates had intermediate sensitivity. Gentamicin was the second most effective drug against these isolates, with $41.94 \%$ sensitive strains. Ciprofloxacin and cefradine were found to be effective antimicrobial drug against these S. aureus strains exhibiting $38.06 \%$ and $32.27 \%$ sensitivity, respectively. Ampicillin demonstrated only $5.26 \%$ sensitivity. The order of resistance of $S$. aureus against 5 groups of antibiotics was ampicillin $(92.10 \%)$, cefradine $(60 \%)$, ciprofloxacin $(59.35 \%)$, gentamicin $(58.06 \%)$ and an intermediate resistance to vancomycin (38.06\%). Cloxacillin resistance for these isolates was 61.29\%. Most of the cloxacillin resistant strains were intermediate resistance towards vancomycin.

\section{MIC \& MBC of clinical isolates of $S$. aureus against five groups of antibiotics}

MIC and MBC were determined for all the $38 \mathrm{~S}$. aureus isolates from clinical samples against five groups of antibiotics, namely penicillins, cephalosporins, aminoglycosides, quinolones and vancomycin (TABLE 2). The MIC obtained for each isolate against various antibiotics and their brands were compared with one another. Kinetics of bactericidal activity showed that the all brands of each antibiotic possessed almost similar activity against clinical isolates of $S$. aureus tested. The MBC value for ampicillin was 2 to 3 two-fold dilution higher than the MIC values. Cephalosporins had MIC range of 8 to $2048 \mu \mathrm{g} / \mathrm{mL}$. MBC values of cefradine and the other brands of the cephalosporins were 2 to 3 two-fold dilution higher than MIC against all isolates. In the current study, bulk of the isolates were resistant to Gentamicin at the concentration $1024 \mu \mathrm{g} / \mathrm{mL}$ to $2048 \mu \mathrm{g} / \mathrm{mL}$ and most of them were inhibited at the range of $4 \mu \mathrm{g} / \mathrm{mL}$ to $512 \mu \mathrm{g} / \mathrm{mL}$ and certainly MIC lies in between for both standard powder and Gentacine. The MBC of gentamicin and gentacine was in range of 32 $\mu \mathrm{g} / \mathrm{mL}$ to $>1024 \mu \mathrm{g} / \mathrm{mL}$.

Staphylococcus aureus is recognized as one of the most important bacterial pathogens contributing towards hospital infection world wide. S. aureus causes localized infection spreading into the blood stream [7] Antibiograms of the isolates, which were collected from $\mathrm{CMOSH}$, had resistance against various antibiotics such as ampicillin, cloxacillin, ciprofloxacin, cholramphenicol, gentamicin, amikacin, and different generations of cephalosporins. Majority of the $S$. aureus strains from the present study were MDR, especially the isolates recovered from pus of the indoor patients of CMOSH. Such MDR pattern among these clinical isolates of $S$. 
aureus has also been documented earlier $[8,9,10]$ who have reported multidrug resistant $S$. aureus as a cause of nosocomial infection during 1992-1997. Methicillin resistant S. aureus were more prevalent in pus (30\%) than blood (24\%) and miscellaneous samples (27\%). Based on results of culture sensitivity, ampicillin was found to be the most resistant (92.1\%), than compared to the other antibiotics. Cloxacillin showed $61.3 \%$ insensitivity towards S. aureus and Vancomycin established 38\% intermediate resistance. Among the 4 studied isolates that were intermediately resistant to Vancomycin were completely resistant to cloxacillin. The comparison of MIC values obtained for each isolate against various antibiotics showed that the all brands of each antibiotic possessed almost similar activity against clinical isolates of S. aureus tested. In the current study, bulk of the isolates was resistant to Gentamicin at the concentration and MIC lies in between for both standard powder and Gentacine. This clearly illustrates emergence and development of resistance against gentamicin by MDR strains of S. aureus. Most of the isolates lie in resistance range of $2 \mu \mathrm{g} / \mathrm{mL}$ to $64 \mu \mathrm{g} / \mathrm{mL}$ with Quinolones, ciprofloxacin in particular. The MBC was 2 to 3 two-fold dilution higher than MIC, which is in concurrence with the works of Brumfitt and Hamilton-Miller [11] and later others [10, 12] documented the similar result.

\section{Tables}

Table 1: List of Antibiotics used with brand name and manufacturers' name

\begin{tabular}{|c|c|c|c|}
\hline SI. No & Group & Antibiotic & Manufacturer \\
\hline \multirow[t]{3}{*}{1.} & \multirow[t]{3}{*}{ Penicillin } & Ampicillin (SP) & Bayer Pharma \\
\hline & & Tycil (L) & Beximco Pharma (Bangladesh) \\
\hline & & Benzapenem (L) & Square Pharmaceuticals Ltd. \\
\hline \multirow[t]{4}{*}{2.} & \multirow[t]{4}{*}{ Cephalosporin } & Cefradine (SP) & Pharmagen Ltd. \\
\hline & & Ceftron $500(\mathrm{~L})$ & Square Pharmaceuticals \\
\hline & & Cefotil $500(\mathrm{~L})$ & Square Pharmaceuticals \\
\hline & & Turbocef $500(\mathrm{~L})$ & Beximco Pharma \\
\hline \multirow[t]{2}{*}{3.} & \multirow[t]{2}{*}{ Aminoglycoside } & Gentamicin (SP) & Eli Lilly \\
\hline & & Gentacine (M) & Hoechst Pvt Ltd. \\
\hline \multirow[t]{3}{*}{4.} & \multirow[t]{3}{*}{ Quniolone } & Ciprofloxacin (SP) & Crosschen International (Switzerland) \\
\hline & & Neofloxcin $500(\mathrm{~L})$ & Beximco Pharma \\
\hline & & Ciprocin $500(\mathrm{~L})$ & Square Pharmaceuticals \\
\hline 5. & Vancomycin & Vancomycin (SP) & Eli Lilly \\
\hline
\end{tabular}

Table 2: MIC and MBC of five tested groups of antibiotics against the Clinical Isolates of S. aureus

\begin{tabular}{|l|l|l|}
\hline Antibiotics used & MIC & MBC \\
\hline Penicillin & .008 to $2.048 \mathrm{mg} / \mathrm{L}$ & $.032 \mathrm{mg} / \mathrm{L}$ to $>1.024 \mathrm{mg} / \mathrm{L}$ \\
\hline Cephalosporin & .008 to $2.048 \mathrm{mg} / \mathrm{L}$ & $.032 \mathrm{mg} / \mathrm{L}$ to $>1.024 \mathrm{mg} / \mathrm{L}$ \\
\hline Aminoglycosides & .004 to $2.048 \mathrm{mg} / \mathrm{L}$ & $.016 \mathrm{mg} / \mathrm{L}$ to $>1.024 \mathrm{mg} / \mathrm{L}$ \\
\hline Quinolones & $.001 \mathrm{mg} / \mathrm{L}$ to $128 \mathrm{mg} / \mathrm{L}$ & $.004 \mathrm{mg} / \mathrm{L}$ to $1.024 \mathrm{mg} / \mathrm{L}$ \\
\hline Vancomycin & $.002 \mathrm{mg} / \mathrm{L}$ to $8 \mathrm{mg} / \mathrm{L}$ & $.004 \mathrm{mg} / \mathrm{L}$ to $.016 \mathrm{mg} / \mathrm{L}$ \\
\hline
\end{tabular}

\section{Conclusion}

This study clearly indicates the trend of $S$. aureus towards development of antibiotic resistance against various antibiotics and even the most effective ones are now becoming insensitive against $S$. aureus. So a more sustainable therapy must be in place to combat $S$. aureus infection. So there are provisions of undertaking a further research to analyze the factors affecting pathogenicity and the interaction with host to find a sustainable solution.

\section{Acknowledgements}

Great indebtedness to the authority of Chattagram Maa Shisu O General Hospital for facilitating this study.

\section{References}

[1] T. Foster and D. Mcdevitt. Surface associated proteins of Staphylococcus aureus: their possible role in virulence, FEMS Microbiol. Lett. 1994; 118: 199-205.

[2] K. Hiramatsu, H. Hanaki, T. Ino, K. Yabuta, T. Oguri, F. Tenover. Methicillin-Resistant Staphylococcus aureus clinical strain with reduced vancomycin susceptibility. J ANTIMICROB CHEMOTHER. 1997, JUL; 40(1):135-6.

[3] Y. Katayama, T. Ito, K. Hiramatsu. A new class of genetic element, staphylococcus cassette chromosome mec, encodes methicillin resistance in Staphylococcus aureus. Antimicrob Agents Chemother, 2000 Jun; 44(6):1549-55.

[4] F. Lowy. Antimicrobial resistance: The example of Staphylococcus aureus. J Clin Invest. 2003; 111(9): 1265-1273.

[5] H. Momtaz, E. Rahimi, and E. Tajbakhsh. Detection of some virulence factors in Staphylococcus aureus isolated from clinical and subclinical bovine mastitis in Iran. African Journal of Biotechnology Vol. 9(25), pp. 3753-3758, 21 June, 2010 
[6] A. Bauer, W. Kirby, J. Sherris. Antibiotic susceptibility testing by a standardized single disc method. Am. J. Clin. Pathol. 996; 45:493-496

[7] F. Espersen. Identifying the patient risk for Staphylococcus aureus blood stream infections. J. Chemotherapy, 1995 7: 11-17.

[8] H. Delancastre, I. Santos, and I. Sanches. Streptococcus pneumoniae: molecular biology and mechanisms of disease. Clinical Microbiology and Molecular Microbiology in alliance CEM/NET. Mary Ann Liebert, Inc., Larchmont, New York, U.S.A. 2000; 451456.

[9] A.Gales, R. Jones, and M. Pfaller,. Two year assessment of pathogen frequency and antimicrobial resistance pattern among organisms isolated from skin and soft tissue infection in Latin American hospitals: results from the sentry antimicrobial surveillance program, 1997-1998. Int. J. Infect. Dis., 2000; 4: 7-84.

[10] S. Raquel, I. Sanches, and D. Dias. Detection of an Archaic Clone of Staphylococcus aureus with Low-Level Resistance to Methicillin in a Pediatric Hospital in Portugal and in International Samples: Relics of a Formerly Widely Disseminated Strain. Journal of Clinical Microbiology, 1999; 37 (6): 1913-1920.

[11] W. Brumfitt, J. Miller. In vitro activity of daptomycin against a world wide collection of methicillin resistant Staphylococcus aureus. Drugs Expt. Clin. Res. 1992; XVIII: 367-369.

[12] R. Connie and M. Geogre.Staphylococcus aureus. Text Book of Diagnostic Microbiology. (Eds.), , WB Saunder, U.K. 2000; $327-$ 337. 